\title{
A Robot as Mobile Sensor and Agent in Data Center Energy Management
}

\author{
Hoi Chan \\ IBM Thomas J. Watson \\ Research Center \\ Hawthorne, NY 10532 \\ hychan@us.ibm.com \\ Jonathan Connell \\ IBM Thomas J. Watson \\ Research Center \\ Hawthorne, NY 10532 \\ jconnell@us.ibm.com

Jeffrey O. Kephart
IBM Thomas J. Watson
Research Center
Hawthorne, NY 10532
kephart@us.ibm.com
Christopher Mansley
Dept. of Computer Science
Rutgers University,
Piscataway, NJ 08854
cmansley@cs.rutgers.edu

\author{
Canturk Isci \\ IBM Thomas J. Watson \\ Research Center \\ Hawthorne, NY 10532 \\ canturk@us.ibm.com
}

ACM Classification Keywords: I.2.9 Computing methodologies, Artificial intelligence, Robotics, Commercial robotics and applications.

General Terms: Design, Management, Performance, Reliability.

Author Keywords: Autonomous systems, self-managing, energy efficiency, data centers, mobile robots.

\section{INTRODUCTION}

In this poster/software demonstration we illustrate the integration of an autonomous mobile robot into a slightly customized version of a commercially available asset and data center energy management application known as Maximo Asset Management for Energy Optimization (MEO), version 7.1.1, through a number of practical scenarios ${ }^{1}$. The scenarios showcase increasing degrees of autonomy and sophistication in the areas of data center mapping, monitoring and thermally-aware diagnostics.

\section{PRIOR WORK}

Hamann et al. [4, 3] pioneered the use of a hand-pushed mobile sensing station, which the authors call the Mobile Measurement Technology (MMT), that was capable of building a data center layout and taking a dense set of sensor readings which could be used as input into a sophisticated set of analytical capability for uncovering data center energy inefficiencies. Examples of such inefficiencies include the identification of under or over-utilized Computer Room

\footnotetext{
${ }^{1} \mathrm{~A}$ paper discussing many of the technical issues involved in the creation of this robot has been separately submitted to this conference. The purpose of this poster and demonstration is to show the robot in action, integrated with visualization and energy management software, much of which is commercially available.
}

Copyright is held by the author/owner(s). ICAC'11, June 14-18, 2011, Karlsruhe, Germany. ACM 978-1-4503-0607-2/11/06.

\author{
Jonathan Lenchner \\ IBM Thomas J. Watson \\ Research Center \\ Hawthorne, NY 10532 \\ lenchner@us.ibm.com \\ Suzanne Mclntosh \\ IBM Thomas J Watson \\ Research Center \\ Hawthorne, NY 10532 \\ skranjac@us.ibm.com
}

Air Conditioning (CRAC) units and the pinpointing of associated hot and cold spots - hot spots arising in areas of under-provisioned cooling and cold spots in areas of overprovisioned cooling. Various authors $[2,1,8]$ have studied the coordinated control of the various knobs available to minimize cooling costs while keeping equipment safe. Patel et al. $[7,6]$ argued for the value of a robotic approach to data center monitoring and management, but without providing any details about an actual implementation. In prior work [5], members of the current author group describe an initial robot for autonomously mapping and monitoring in data centers - in other words, a robot for taking on much of the role of the human in the hand-pushed MMT solution of Hamann et al. In the current work, we take the robot several steps further, first by making the robot far more robust to practical environments and second by taking steps towards imbuing the robot with automated, thermally-aware, diagnostic capability.

\section{ROBOT AS MOBILE SENSOR}

In its most fundamental role, we showcase the robot being placed in an unknown data center and autonomously mapping out the data center and taking a series of temperature and humidity readings. In this role the robot behaves like a mobile sensor, feeding its data into IBM Tivoli Monitoring for Energy Management (ITMfEM) and from there into MEO. As the layout and thermal profile are being generated, the user can observe their dynamic evolution, along with the ever-changing location of the robot. For example, in Figure 1 we see an evolving layout and thermal profile after the robot has explored a new data center for just 15 minutes. In addition, all data collected by the robot are warehoused by ITMfEM and MEO for possible subsequent analysis.

\section{ROBOT AS AGENT}

We illustrate the robot as agent in two additional scenarios, showcasing various degrees of agency/autonomy:

(1) In a previously scanned data center, we, a human data center administrator, notice a thermal anomaly developing, 
based on readings from a smattering of static sensors attached to racks. We dispatch the robot to probe the area of concern and in so doing the robot collects nearby sensor readings sufficient to get a fine-grained thermal profile of the area of concern. It also takes still pictures in the vicinity of the emerging thermal problem. The fine grained thermal profile and still images are then available to a data center administrator through the MEO graphical user interface. See Figure 1.

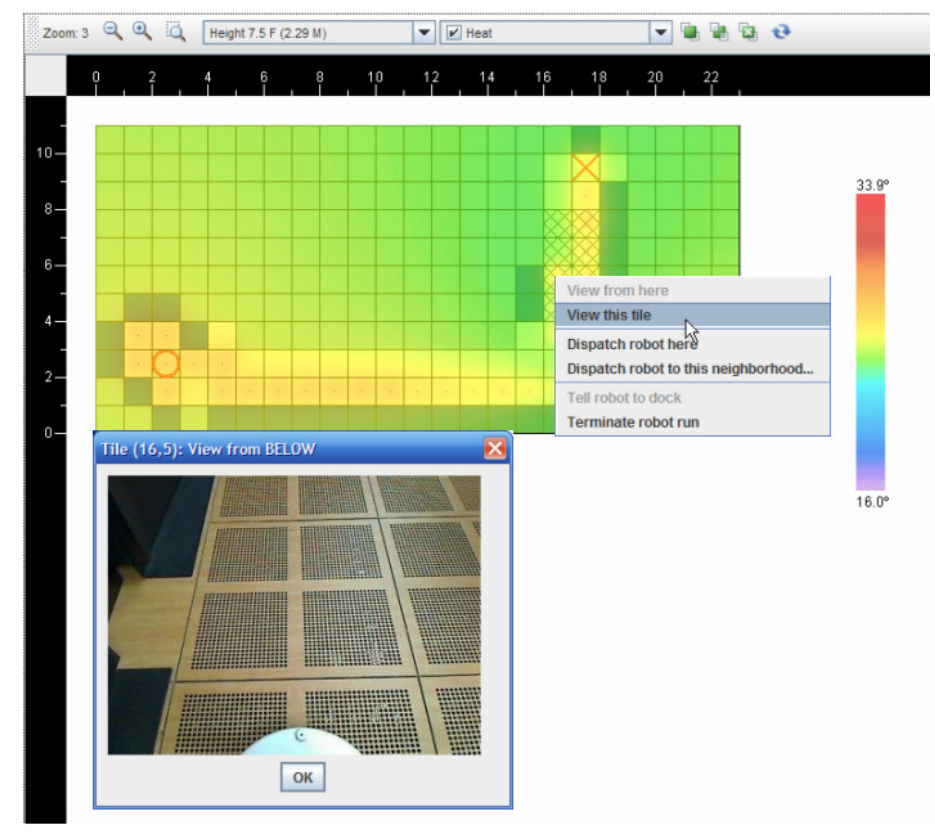

Figure 1: Inside the MEO tool, a user examining the image of a perforated tile in a data center. Such tiles allow for the venting of cool air into the raised floor area. The robot is only about 15 minutes into its scan of this particular data center. The "O" indicates where the robot started from, while the " $\mathrm{X}$ " indicates where the robot is now. Redder colors in the "heat map" indicate warmer temperatures. The actual key mapping colors to temperatures is given at the far right. The cross-hatched squares indicate the locations of perforated tiles. Dark squares indicate the presence of obstacles, like server racks, air conditioning units, or walls.

(2) In a data center in which the robot has done a prior dense scan, the robot chooses a set of most informative locations from which to do faster, selective scans as described in [5]. Within the MEO application, hot thresholds have been set $^{2}$ in the neighborhoods of the front of various racks to help ensure sufficiently cool air is received into the air intake of the various IT equipment. In one of the robot's selective scans, hot threshold violations are detected in the ambient air in the vicinity of one of these racks and an emerging thermal problem is noted. The robot issues an alert that is surfaced through ITMfEM and MEO and the software autonomously notifies the robot to do a finer-grained, localized, scan and take images, along the lines of (1).

\footnotetext{
${ }^{2}$ The application also allows for the setting of cold thresholds, which, if exceeded, indicate the excessive use of cooling.
}

\section{REFERENCES}

[1] T. Boucher, D. Auslander, C. Bash, C. Federspiel, and C. Patel. Viability of dynamic cooling control in a data center environment. In Proc. of the 9th Int'l Conf. on Thermal and Thermomechanical Phenomena in Electronics Systems (ITHERM), pages 445-452, Las Vegas, NV, August 2004.

[2] R. Das, H. Hamann, J. Kephart, and J. Lenchner. Utility-function-driven energy-efficient cooling in data centers. Proceedings of the 7th International Conference on Autonomic Computing (ICAC), pages 61-70, 2010.

[3] H. Hamann, T. van Kessel, M. Iyengar, J.-Y. Chung, W. Hirt, M. A. Schappert, A. Claassen, J. M. Cook, W. Min, Y. Amemiya, V. Lopez, J. A. Lacey, and M. O'Boyle. Uncovering energy efficiency opportunities in data centers. IBM Journal of Research and Development, 53(3):10:1-10:12, 2009.

[4] H. F. Hamann, M. Schappert, M. Iyengar, T. van Kessel, and A. Claassen. Methods and techniques for measuring and improving data center best practices. In Proceedings of 11th Intersociety Conference on Thermomechanical Phenomena in Electronic Systems, pages 1146-1152, May 2008.

[5] C. Mansley, J. Connell, C. Isci, J. Lenchner, J. O. Kephart, S. McIntosh, and M. Schappert. Robotic mapping and monitoring of data centers. IEEE International Conference on Robotics and Automation (ICRA), 2011.

[6] C. Patel. A vision of energy aware computing from chips to data centers. Proc. of the International Symposium on Micro-Mechanical Engineering (ISMME), Dec 2003.

[7] C. Patel, C. Bash, R. Sharma, A. Beitelmal, and R. Friedrich. Smart cooling of datacenters. Proc. of the PacificRim/ASME Int'l Electronics Packaging Tech. Conference and Exhibition (IPACK), July 2003.

[8] R. Raghavendra, P. Ranganathan, V. Talwar, Z. Wang, and X. Zhu. No power struggles: A unified multi-level power management architecture for the data center. In International Conference on Architectural Support for Programming Languages and Operating Systems (ASPLOS), 2008. 